\title{
Rudolf von Jhering und die Freude am Rechtsfall
}

I.

Will man sich anlässlich seines 200. Geburtstages der herausragenden Leistungen Rudolf von Jherings bewusst werden, so kann dies nicht ohne den einen Blick auf seine bislang viel zu wenig beachtete "Rolle als Reformator des Rechtsunterrichts" erfolgen. Diese Stellung ist maßgeblich durch zwei Fallsammlungen begründet, die - seinerzeit eine Novität - Jhering für den akademischen Unterricht zusammenstellte und die entscheidend zur anschaulichen Vermittlung des Rechtsstoffes und zu kontrollierter rechtspraktischer Lösung von Rechtsfällen im juristischen Studium beitrugen. Eine Betrachtung beider Kollektionen dürfte sich deshalb anbieten.

Im Jahre 1847 brachte Jhering als Ordinarius in Rostock ein Heft von 170 Seiten heraus mit dem Titel 'Civilrechtsfälle ohne Entscheidungen' ${ }^{1}$. Es enthält 100 von ihm selbst entwickelte oder zusammengetragene Rechtsfälle und im Anhang 36 weitere des verstorbenen Georg Friedrich Puchta, dem wissenschaftlichen Idol Jherings. Gewidmet hat Jhering das Heft Heinrich Thöl, einem seinerzeit berühmten Handelsrechtler ${ }^{2}$, dem er in der Begründung der Widmung "mit dankbarer Anerkennung" bekundet:

\footnotetext{
"Als ich vor einem Decennium nach Göttingen kam, um das Studium der Rechtswissenschaft dort fortzusetzen, ward mir von allen Seiten ein von I h n e n gehaltenes s. g. [so genanntes] PandektenPraktikum dringend anempfohlen. Wie sehr es mich freut, diese Weisung befolgt zu haben, glaubte ich I h n e n nicht besser zeigen zu können als durch das öffentliche Bekenntniß, daß ich erst durch $\mathrm{S}$ i e die Jurisprudenz liebgewonnen habe, erst durch $\mathrm{S}$ i e zum juristischen Denken angeleitet worden bin. [-] ... drängt es mich, die Herausgabe eines Werkes, das zu dem eben erwähnten Collegium in naher Beziehung steht, dazu zu benutzen, [-] I h n e n öffentlich die Versicherung zu geben, daß ich meine juristische Bildung erst vom Besuch I h r e r Vorlesung an datire ..."
}

Dass Jhering die Augen für eine anschauliche Erörterung des Rechtsfalles gerade von einem Handelsrechtler wie Thöl geöffnet worden sind, wird auch damit zusammenhängen, dass dieser durch sein Fach in besonderem Maße mit rechtspraktischen Fragen befaßt war und

$\mathrm{Zu}$ akademischen Zwecken herausgegeben von R. Jhering, Leipzig, Breitkopf und Härtel, 1847. 6. März 1807 (Lübeck) - 16. Mai 1884 (Göttingen). 


\section{R. KNÜTEL}

dass für sein Werk "die gründlich civilistische Behandlung der Handelsrechtsinstitute" charakteristisch geworden ist. ${ }^{3}$

II.

2.1.

Im Vorwort der "Civilrechtsfälle" hebt Jhering eingangs hervor: "Welch' ein wichtiges Hülfsmittel zur Förderung des akademischen Studiums der Pandekten die Entscheidung von Rechtsfällen gewährt, scheint noch wohl nicht gehörig beherzigt zu sein", und er führt dann zunächst zwei Vorteile eines Civil- oder Pandekten-Praktikums an, erstens den, dass Fallerörterungen für den Lehrer wie für den Zuhörer einen Test bilden, den Erfolg der in den theoretischen Vorlesungen vermittelten Kenntnisse zu beurteilen und den Stand des erlangten Wissens zu überprüfen, und zweitens den mnemotechnischen Aspekt, dass sich die abstrakten Rechtssätze dem Anfänger bekanntlich nicht leichter und fester einprägen als gerade am konkreten Fall. Vor allem aber betont Jhering die besonderen Schwierigkeiten, die sich aus dem abstrakten Charakter der Jurisprudenz ergeben:

\footnotetext{
"Kein Studium, etwa das der Mathematik und reinen Philosophie ausgenommen, möchte eben wegen jenes Requisites bei seinem Beginn so schwierig und darum so wenig anziehend sein, als das der Jurisprudenz. Der Anfänger sieht sich hier gewissermaaßen in eine ganz fremde Begriffs-Welt versetzt, die keine Anknüpfungspunkte mit seiner bisherigen Bildung darbietet ... Es wird die Anforderung an ihn gestellt, sich sicher auf diesem abstrakten Boden zu bewegen ...; und doch fehlt es ihm an der wesentlichsten Voraussetzung, ... nämlich an der Fähigkeit des abstrakten Denkens." ... Abhilfe "wird nun am sichersten dadurch erreicht, daß man dem Anfänger das Abstrakte anfänglich nicht in seiner reinen, nackten Form vorführt, sondern in seiner Verkörperung im Rechtsfall." ...4
}

Im weiteren weist Jhering auf die "ihm sehr fühlbare" Schwierigkeit hin, seine Fallsammlung zusammenzubringen, "nämlich der Mangel an brauchbaren Rechtsfällen". Ungeachtet der reichen Literatur von Rechtssprüchen, Gutachten u.s.w. sei seine Ausbeute höchst dürftig gewesen, und er habe alle brauchbaren Rechtsfälle, die ihm namentlich durch Mitteilung von praktischen Juristen zur Kenntnis kamen, gesammelt, um daraus eine Auswahl zu treffen. Zugleich bittet er, "alle, die im Stande und nicht abgeneigt sind, das Unternehmen mit passenden Beiträgen zu unterstützen, ihm dieselben für die späteren Hefte zu überlassen"5.

3 C. Gareis, Das Deutsche Handelsrecht, 4. Aufl. Berlin 1892, S. 39, 43 zu H. Thöl, Handelsrecht, Bd. 1, Göttingen 1841 (Handelsrechtsinstitute), 6. Aufl. 1878; Bd. 2, 1848 (Wechselrecht; in "seiner an Bedeutung alle anderen überragenden Bearbeitung", C. S. Grünhut, Wechselrecht, Bd. 1, Leipzig 1897, S. 112, vgl. S. 265), 4. Aufl. 1878; Bd. 3, 1880 (Das Transportgewerbe).

4 Vorwort S. VII - IX, ganz ähnlich noch die 12. Aufl. 1913, S. V/VI.

5 Vorwort S. XII. Ungeachtet der Zusage angemessener Honorierung hat die Bitte offenbar wenig Widerhall gefunden. 


\section{RUDOLF VON JHERING UND DIE FREUDE AM RECHTSFALL}

Abschließend gibt Jhering Hinweise auf die Auswahl der von ihm aufgenommenen Fälle, die in der bei weitem größeren Mehrzahl aus mündlichen Mitteilungen entstanden seien, sowie auf die Darstellung, bei der er es sich zur Aufgabe gemacht habe, nie seine eigene Ansicht durchschimmern zu lassen.

\section{2.}

Jhering hatte ein Desiderat der juristischen Ausbildung erkannt; seine 'Civilrechtsfälle' wurden ein großer Erfolg. Zwar kam eine 2. wesentlich veränderte Auflage erst 1870 heraus, dann erschienen jedoch in schnellerer Abfolge 1876 die 3., 1881 die 4., 1888 die 5. und 1892 die 6. und zugleich letzte noch von Jhering herausgegebene Bearbeitung. Die 7. Aufl. war vermutlich ein bloßer Nachdruck der 6. 1897 kam dann die 8. von Ferdinand Regelsberger mit Rücksicht auf das Bürgerliche Gesetzbuch bearbeitete Auflage heraus. Er war Herausgeber auch der 9. bis 11. Auflage von 1909. Die 12. und letzte Auflage von 1913 wurde von Theodor Kipp mit kleinen Änderungen versehen; als Schüler Windscheids hatte er zuvor die 8. und die 9. Aufl. von dessen so maßgeblich gewordenem Lehrbuch des Pandektenrechts herausgebracht ${ }^{6}$.

\section{III.}

\section{1.}

Sehr bemerkenswert ist, dass aus den Civilrechtsfällen ein weiteres Werk erwachsen ist: Für die 2. Aufl. der Civilrechtsfälle arbeitete Jhering seine praktischen Fälle weitgehend um und setzte an die Stelle der 36 Rechtsfälle Puchtas, welche die zweite Abteilung gebildet hatten, kleine anschauliche Fälle und Fragen unter dem Titel 'Die Jurisprudenz im täglichen Leben'7. Diese neue 2. Abteilung wurde ab der 4. Aufl. aus den Civilrechtsfällen ausgegliedert und unter dem Titel 'Jurisprudenz des täglichen Lebens. Eine Sammlung an Vorfälle des gewöhnlichen Lebens anknüpfender Rechtsfragen' (im folgenden 'Jurisprudenz') erstmals 1886 selbständig als 6 . Auflage ${ }^{8}$ publiziert.

6 Schon Jhering hatte für die 5. Aufl. als Mitarbeiter die Professoren Regelsberger, Kipp, Lenel, Goldschmidt und (später noch) Detmold gewonnen; unter Regelsberger war Andreas von Tuhr hinzugekommen und ein Landgerichtsdirektor Eller, der zahlreiche Fälle vorlegte. Durch das Inkrafttreten des BGB waren manche Fälle auszutauschen oder zu verändern; auch war der Bestand zu erweitern, so dass aus dem Heft von ursprünglich 170 S. in der letzten Auflage 320 S. geworden waren. - Stichworte zu den Auflagen 1 - 5 und den äußeren Veränderungen finden sich in der Bibliographie Rudolf von Jherings von M. Losano, in: F. Wieacker, Chr. Wollschläger (Hrsg.), Jherings Erbe. Göttinger Symposion zur 150. Wiederkehr des Geburtstages von Rudolf von Jhering, Abh. AdW Göttingen, Phil.-Hist. Kl. III Nr. 75, Göttingen 1970, S. 256-275.

$7 \quad$ S. $201-230$ der 2. Aufl.; vgl. Losano S. 264.

8 Das Ausgangswerk, die Civilrechtsfälle, war erst 1888 zur 5. Aufl. gediehen; vermutlich sollte diese Auflage zwei Jahre früher erscheinen. Auch Jhering litt bekanntlich unter Terminnöten. 


\section{R. KNÜTEL}

\section{2 .}

Auch diesem Werk war anhaltender Erfolg beschieden: Eine 7., wesentlich vermehrte Auflage konnte schon 1889 erscheinen, eine 8. noch von Jhering selbst besorgte 1892, die 11 . wurde 1898 von dem großen Romanisten Otto Lenel herausgegeben, die 12. (1902/1903) und 13. (1908) von G. Detmold (Professor in Göttingen) und schließlich die 14. (1921/1922) und 15. (1927) von dem bedeutenden Zivilisten Paul Oertmann. Insgesamt sind damit neun Ausgaben dieses Werkes erschienen.

\section{3.}

Die 'Jurisprudenz' ist nach verschiedenen Lebens- oder Sachbereichen in 21 Abschnitte gefasst, in der 7. Aufl. etwa beginnend I. Nach und auf der Eisenbahn, II. Im Gasthof, III. In der Miethswohnung, IV. Aus dem häuslichen Leben ... IX. Ein Hausbau, X. Im nachbarlichen Verhältniß, XI. Auf der Straße, XII. In der Noth, XIII. Aus dem geselligen Leben ... XX. In der Fremde sowie schließlich XXI. Allerlei. In der Regel werden in diesen Abschnitten Ausgangssituationen geschildert, zu denen dann mehrfach abgewandelt verschiedene Fragen aufgeworfen werden. Nehmen wir als Beispiel sogleich den Fall der Abteilung I Nr. 1-4, der offenbar in allen Auflagen am Anfang des Werkes stand, und geben in Stichworten Hinweise auf Regelungen oder Erwägungen, die sich nach dem BGB auf der Grundlage der herrschenden Meinung anbieten.

1. Um nach der Eisenbahn zu fahren, rufen wir den an unserer Wohnung vorbeifahrenden Omnibus an und steigen ein, ohne ein Wort zu verlieren. Hat dies Einsteigen eine juristische Bedeutung und welche? Welches Rechtsgeschäft wird dadurch abgeschlossen? [Ein gut zu diskutierender Ausgangspunkt: Ist der Bus ein "rollendes Angebot" oder der Fahrgast der Offerent? Spielen besondere Umstände eine Rolle, wie Lösen der Fahrkarte ${ }^{9}$ beim Fahrer oder freier Zutritt, Kontrahierungszwang etc.? Beförderungsvertrag]

2. Hat auch das Einsteigen in den Wagen eines Freundes, der uns zu einer Spazierfahrt abholt, eine juristische Bedeutung? [Bloßes Gefälligkeitsverhältnis oder stillschweigende Kausalabrede über Unentgeltlichkeit?] Worin liegt der Unterschied beider Fälle?

3. Welche rechtliche Stellung nimmt im obigen Fall der Conducteur des Wagens ein? [Vertreter, $\S \S$ 164 ff. BGB; Erfüllungsgehilfe, § 278 BGB] Haftet die Omnibusunternehmung bloß aus dem von uns mit ihm abgeschlossenen Contract oder auch aus dessen Delikten, z. B. wenn derselbe eine von uns vergessene Reisetasche unterschlagen oder uns wissentlich zu wenig Geld herausgegeben hat? [Ja, nach $\S 831$ mit $\S 823$, oder mit $\S 826$ BGB, zudem: strafbare und vorsätzliche Handlung nur "bei Gelegenheit" der Erfüllung?].

4. Auch, wenn letzteres unwissentlich geschehen ist? [Ja; der Verrichtungsgehilfe des $\S 831$ braucht nicht schuldhaft zu handeln].

9 Dafür Jhering, Culpa in contrahendo, Jherings Jahrbücher 4 (1861) 96. 


\section{RUDOLF VON JHERING UND DIE FREUDE AM RECHTSFALL}

Bisweilen ergeben sich recht überraschende Zusatzfragen. So beginnt in der 7. Auflage v. 1889 in Abt. X (Im nachbarlichen Verhältniß) der zweite Fragenkomplex:

2. Unser Obst fällt von den hinüberhängenden Zweigen in den Garten des Nachbarn; muß letzterer dulden, daß wir es uns holen? [§911 BGB: Der Nachbar wird Eigentümer hinüberfallender Früchte.] ... 5. Ist er dadurch, daß das Obst auf seinem Grundstück liegt, Besitzer des Obstes geworden? [wenn er Besitzwillen hat] 6. Ist dasselbe, bis wir unsererseits es einsammeln, herrenlos? [§ 911]. 7. Muß der Nachbar dulden, daß unsere Zweige hinüberhängen? [§ 910 S. 2: Er kann nach erfolgloser Fristsetzung abschneiden.] ... 9. Der Nachbar hat sich einen auf unser Grundstück gefallenen Meteorstein angeeignet; können wir ihn vindizieren? ... Er hat ihn an das naturhistorische Kabinet für 200 Mark verkauft; können wir den Betrag in Anspruch nehmen?

Es zeigt sich, dass das BGB den hier wiedergegebenen (sowie weggelassenen) Fragen zum guten Teil die Schwierigkeit genommen hat ${ }^{10}$, abgesehen vom Fall des Meteoriteneinschlags, einem für die Jurisprudenz des täglichen Lebens eher seltenen Ereignis ${ }^{11}$, das allerdings juristisch interessante Fragen auslösen kann. Wir greifen dazu auf einen "Fall aus dem Leben" zurück:

Am 6.4.2002 ging über Südbayern ein Meteorit nieder, der in knapp 22 Kilometer Höhe zerplatzte. Ein erstes Teilstück wurde im Juli 2002 nahe des Schlosses Neuschwanstein gefunden ("Neuschwanstein 1" genannt), ein zweites im Mai 2003 im Ammerseegebirge ("Neuschwanstein 2") und ein drittes größtes, gut 2,8 kg schweres ("Neuschwanstein 3") Ende Juni 2003 schließlich von einem Physiker P am Tiroler Altenberg auf einer Geröllhalde, die im Eigentum der österreichischen Gemeinde Reutte (= R) steht.

Die R verklagte den P auf Herausgabe. Das Landgericht Augsburg ${ }^{12}$ wies die Klage ab. Nach dem einschlägigen österreichischen ABGB sei R weder durch "Zuwachs" (Alluvion) Eigentümerin nach $\S 411$ ABGB (auch nicht in analoger Anwendung) geworden noch aufgrund einer Zueignung gemäß $\S \S 380,381$ ABGB, weil dafür ein genereller Besitzwille hinsichtlich aller Sachen, die sich auf den eigenen Liegenschaften befinden, ohne erkennbare tatsächliche Erwerbshandlungen nicht genüge. Auch scheide ein Erwerb gleichteiligen Miteigentums am Meteoritenfragment in analoger Anwendung der Vorschriften über den Schatzfund ( $\S 398,399$ ABGB) aus. Zwar lasse sich der Begriff des Schatzes auch auf ursprünglich herrenlose Sachen von Wert ausdehnen, doch habe der Meteorit offen

10 Jedenfalls seit der 13. Aufl. v. 1908 wird im Fall X.2 nur nach einer Duldungspflicht nach römischem Recht und im Fall X.7 (13. Aufl. X.4) nach römischem Recht und nach dem BGB gefragt.

11 Während Jherings Leben sind für Bayern wohl nur zwei Fälle bekannt, 1846 bei Schönenberg und 1869 bei Krähenberg.

12 LG Augsburg, Urteil v. 6.7.2007 (Geschäftszeichen 8 O 1758/06). 


\section{R. KNÜTEL}

zu Tage gelegen, nicht "im Verborgenen" und bei knapp 15 Monaten auch nicht "lange". Nachdem R Berufung eingelegt hatte, verglichen sich die Parteien vor dem Oberlandesgericht. P übernahm eine Ausgleichszahlung und die Gerichtskosten und behielt den Asteroiden.

Alldem ist zuzustimmen und auch für das deutsche Recht im Einzelfall eine analoge Anwendung der Schatzfundregel des $§ 984$ BGB nicht grundsätzlich auszuschließen. Inzwischen hat sich Rechtslage allerdings dadurch wesentlich geändert, dass in nahezu allen deutschen Bundesländern ein Schatzregal eingeführt worden ist, wonach Funde/Fundstücke von besonderer kultureller oder wissenschaftlicher Bedeutung sogleich in das Eigentum des Bundeslandes fallen, gleichgültig ob es auf öffentlichem oder privatem Grund zu dem Fund kam. In Bayern gilt dies jedoch nicht, so dass Jherings Fall dort seinen juristischen Reiz behalten hat - und immerhin soll es in Bayern mittlerweile zwei weitere Meteoriteneinschläge gegeben haben ${ }^{13}$.

Für Jherings Fall X 9 würde sich nach alldem folglich in Bayern ergeben: Da der Eigentümer noch keine Kenntnis davon hatte, dass der Meteorit auf sein Grundstück gestürzt war, und deshalb auch keinen Besitzwillen hinsichtlich des Meteoriten haben konnte und da auch ein genereller Besitzerwerbswille im Hinblick auf Meteoriteneinschläge nicht zu unterstellen ist, hat der Nachbar mit der Begründung des Eigenbesitzes die res nullius erworben, § 958 $\mathrm{BGB}^{14}$.

IV.

Jherings Leistung und ihre Wirkung auf die juristische Ausbildung wird verständlich, wenn man sich den juristischen Gehalt seiner Rechtsfälle vergegenwärtigt. Wir wollen dies mit der Erörterung von fünf Fällen versuchen, die im Sachgehalt recht kurz sind, deren Lösung nicht von Besonderheiten des römischen Rechts abhängt ${ }^{15}$ und in denen Jherings Strategie deutlich wird:

"Der Rechtsfall muß schon als solcher dem Studierenden ein gewisses Interesse abnötigen, d.h. das Faktische desselben muß so beschaffen sein, daß es auch für den Unkundigen einen gewissen Reiz darbietet, daß der Fall sich dazu eignet, erzählt und besprochen zu werden, und durch seine Anschaulichkeit und individuelle Gestaltung sich dauernd dem Gedächtnis einprägt." 16 


\section{RUDOLF VON JHERING UND DIE FREUDE AM RECHTSFALL}

4.1.

Ein Krönungszug (Civilrechtsfälle 1847 Nr. LXX)

Der Fall findet sich von der 1. bis in die letzte Auflage und ist zu einem Standardfall gewor$\operatorname{den}^{17}$.

Bei einer bevorstehenden Rückkehr des Königs von Preußen nach Berlin war ein feierlicher Empfang desselben von Seiten der Stadt beabsichtigt, und der Tag des Einzuges auf den 15. September festgesetzt. Mit Rücksicht hierauf mietheten sehr viele Personen in den Straßen, durch die der Zug gehen sollte, Zimmer und Fenster auf jenen Tag. Später erfuhr man, daß der Einzug erst am 17. Septbr. Statt finden würde. Viele von den Vermiethern verlangten trotzdem den für den 15. September veraccordirten Miethzins und vermietheten auf den 17. anderweitig. Sind sie zu beidem berechtigt?

Dem Fall liegt zugrunde, dass der Empfang von Friedrich Wilhelm IV. um zwei Tage verschoben werden musste, als dieser von seiner Huldigungsreise, die er nach Antritt der Regierung 1840 unternommen hatte, nach Berlin zurückkehrte. Es liegt nahe, dass sich in Monarchien derartige Begebenheiten wiederholen, und in der Tat haben entsprechende Fälle die englischen Gerichte beschäftigt. Leading case ist Krell v. Henry ${ }^{18}$. Aus Anlass der Krönung von Edward VII. sollten 1902 am 26. Juni eine "Coronation Procession" vom Buckingham Palace nach Westminster und am Tag darauf ein "Royal Progress" durch die City stattfinden. Der Beklagte hatte für beide Tage eine licence zum Gebrauch der Wohnung des Klägers auf der Pall Mall, die Teil der Strecken war, erworben. Zwei Tage vor der Zeit mussten die Umzüge wegen einer Erkrankung des Königs abgesagt werden. Vor dem Court of Appeal unterlag der "Vermieter" mit seiner Zahlungsklage.

Die "'Krönungsfälle' des traditionsfrohen England" ${ }^{19}$ wurden in der deutschen Zivilrechtslehre zum "Paradefall"20 der Lehre von der Geschäftsgrundlage, die nach gut 150 Jahre währender Diskussion durch das Gesetz zur Modernisierung des Schuldrechts seit 2001 in $\S 313$ BGB gesetzliche Anerkennung gefunden hat. Danach kann, wenn sich die Umstände, die zur Grundlage des Vertrags geworden sind, schwerwiegend verändert haben und die Parteien bei Kenntnis den Vertrag nicht oder mit anderem Inhalt abgeschlossen hätten, An-

17 1. Aufl. 1847, S. 120; 12. Aufl. 1913, S. 85 f. Nr. 45 (wo aus dem König in Preußen das "jung vermählte Kronprinzenpaar" geworden und die Vermietung "für hohe Preise" erfolgt ist).

18 [1903] 2 Law Reports, King's Bench Division 740, dazu R. Zimmermann, "Heard melodies are sweet etc.", AcP 193 (1993) $141 \mathrm{f}$.

19 F. Wieacker, Gemeinschaftlicher Irrtum der Vertragspartner etc., in: F. Wieacker, Kleine juristische Schriften, Göttingen 1988, S. 199 (aus Fschr. W. Wilburg, 1965, S. 229, 231).

20 So z. B. K. Larenz, Geschäftsgrundlage und Vertragserfüllung, 3. Aufl. München 1963, S. 47, 74f.; W. Flume, Allgemeiner Teil des Bürgerlichen Rechts, Bd. II (Das Rechtsgeschäft), 4. Aufl., Berlin, Heidelberg 1992, S. 498 (der selbst diese Fälle als solche nachträglicher Unmöglichkeit einordnet); P. Oertmann, Die Geschäftsgrundlage, Leipzig 1921, S. 149 ("in dem bekannten Fall bei Ihering"); R. MeyerPritzl in HKK (Histor.-krit. Kommentar zum BGB, hgg. von M. Schmoeckel, J. Rückert, R. Zimmermann), Bd. II/2, Tübingen 2007, §§ 313 - 314, Rz. 62, S. 1747. 


\section{R. KNÜTEL}

passung verlangt werden oder, wenn dies nicht möglich oder zumutbar ist, der benachteiligte Teil vom Vertrag zurücktreten. Nach heutigem Recht könnten in Jherings Fall die Mieter also, wenn ihnen eine Verschiebung auf den späteren Termin nicht zumutbar wäre, zurücktreten; den Vermietern stünde es dann frei, anderweitig zu vermieten.

Die Entwicklung im deutschen Recht ist im Ergebnis mithin von der englischen Rechtsprechung beeinflusst. Diese war zu ihrem Ergebnis über die Konstruktion gelangt, die Durchführung der Krönungszüge bilde eine "implied condition" des Vertrages, und es war klar, dass damit zugleich über die Lehre von der "frustration of contract" römisches Recht angewendet wurde ${ }^{21}$. Es besteht der Unterschied, dass nach englischem Recht der Vertrag mit Ausfall der stillschweigenden Bedingung eo ipso aufgelöst wird, nach der Lehre von der Geschäftsgrundlage in $§ 313$ BGB 2001 dagegen erst nach Rücktritt.

Noch während des Prozesses Krell v. Henry hatte Alfred Manes von dem Fall und juristischen Vorüberlegungen des Gerichts berichtet und gemeint, jedenfalls würden "die Verschiebung der Krönung König Edwards VII. und ihre privatrechtlichen Folgen als ein gutes Schulbeispiel auch deutschen Rechtsbeflissenen interessant sein"; Heinrich Titze wies demgegenüber darauf hin, "dass wir in Deutschland einen gleichen Rechtsfall erlebt haben" und "dieses 'Schulbeispiel' ..., namentlich seitdem es Jhering in seine berühmte Sammlung von Civilrechtsfällen ohne Entscheidungen aufgenommen hat, ... schon vor dem englischen Rechtsfall Jahrzehnte hindurch auf unseren Universitäten von den Jüngern des Rechts bearbeitet worden" ist ${ }^{22}$. Und wir sind beeindruckt von Jherings Gespür für reizvolle Rechtsfälle, das um so beachtlicher ist, weil der Fall ungeachtet der Regelung in $\S 378$ I 5 ALR $^{23}$ in der preußischen Rechtsliteratur wohl kaum Beachtung gefunden haben dürfte ${ }^{24}$. Die von Augustin von Leyser erneuerte Lehre von der clausula rebus sic stantibus war Jhering selbstverständlich bekannt ${ }^{25}$.

21 R. Zimmermann (Fn. 18) 141 f., der die einleitende Bemerkung von Vaugham Williams, L. J. im Urteil Krell v. Henry (Fn. 18) 747 f. zitiert: "The real question in this case is the extent of the application in English law of the principle of the Roman law, which has been adopted and acted on in many English decisions".

22 A. Manes, Englische Königskrönung und Privatprozesse, DJZ 7 (1902) 526; H. Titze, DJZ (1902) 575 (unter demselben Titel). Auch Th. Rüfner weist auf diese Diskussion hin in N. Jansen, R. Zimmermann (Hg.), Commentaries on European Contract Law, Oxford 2018, zu Art. 6: PECL Rz. 11 Fn. 48 (im Druck).

23 "Wird jedoch durch eine solche unvorhergesehene Veränderung [sc. der Umstände] die Erreichung des ausdrücklich erklärten, oder aus der Natur des Geschäfts sich ergebenden Endzwecks beyder Theile unmöglich gemacht, so kann jeder derselben von dem noch nicht erfüllten Vertrage wieder abgehn."

24 Weder C. F. Koch, Das Recht der Forderungen nach Gemeinem und nach Preußischem Rechte, Bd. 2, 2. Aufl. Berlin 1859, S. 505 ff. noch H. Dernburg, Das Obligationenrecht Preußens und des Reichs, 4. Aufl. Halle 1889, S. 253 ff. beachten den Fall.

25 Leyser, Meditationes ad Pandectas, Spec. 40, med. IV (Bd. 1, Frankenthal 1778, S. 411): Omne pactum, omnis promissio, rebus sic stantibus intelligenda est. - Windscheids Lehre von der Voraussetzung lag 1847 noch nicht vor (1850). 


\title{
RUDOLF VON JHERING UND DIE FREUDE AM RECHTSFALL
}

Anzumerken ist schließlich, daß die Krönungsfeierlichkeiten von Edward VII am Ende auch in Jherings Sammlung zum Fall wurden: Außer den beiden genannten Umzügen sollte am 26. Juni, dem dritten Tag der Festlichkeiten, bei Spithead eine Parade (review) der Kriegsflotte veranstaltet werden; doch mußte sie ebenfalls abgesagt werden. Zu ihrer Besichtigung mit zahlenden Gästen hatte Mr. Hutton ein Dampfboot gemietet, und auch hier kam es zum Streit um die Zahlungspflicht und in der 13. Auflage der 'Jurisprudenz' von 1908 zur Aufnahme eines neues Falles:

\begin{abstract}
"Der norddeutsche Lloyd machte bekannt, daß er zu einer an bestimmtem Tage bei Spithead (Reede vor dem Kriegshafen Portsmouth) stattfindenden Flottenrevue einen Dampfer entsenden werde. Der Dampfer fuhr rechtzeitig mit seinen Passagieren nach Spithead, da aber infolge unvorhergesehener Umstände die Revue um einige Tage hinausgeschoben und der Dampfer in Bremen ... gebraucht wurde, fuhr der Kapitän trotz der Bitten der Passagiere ... zurück. Können die Passagiere ihr Fahrgeld zurückverlangen? Steht der Fall demjenigen gleich, wenn für die Besichtigung eines Festzugs ein Fenster zu hohem Preis vermietet, der Festzug nachher aber abgesagt oder verlegt wurde?"26
\end{abstract}

4.2.

Ein mildtätiger ehemaliger Waisenknabe (Jurisprudenz 1889, Nr. XXI. 15)

Ein ehemaliger Waisenknabe, der im Laufe der Zeit ein reicher Mann geworden ist, hat die Gewohnheit, an seinem Geburtstage die sämtlichen Waisenkinder der Waisenanstalt zu sich in seine Villa einzuladen. Dort werden sie zuerst bewirtet, dann erfolgt ein Suchen nach den in seinem Park versteckten Gegenständen: hartgesottenen Eiern, Obst, Spielsachen, selbst einigen Uhren, die demjenigen zufallen, der sie findet. Wie ist der Vorgang juristisch zu bestimmen: als Auslobung, Occupation herrenloser Sachen, Tradition an eine persona incerta? Angenommen, es hätte sich unter die Waisenkinder ein nicht zu ihnen gehöriges Kind gemischt, oder es würde einer der liegen gebliebenen Gegenstände später von einem der im Park beschäftigten Arbeitsleute entdeckt, würde auch ihnen die gefundene Sache zufallen? Sollte diese Frage im Sinne der Absicht des Urhebers zu verneinen sein, was ergibt sich daraus für den juristischen Charakter des obigen Vorganges?

Eine Auslobung war nach überwiegender und von Jhering geteilter Ansicht das öffentliche Versprechen einer Leistung an denjenigen, der irgendwie gemäß der im Versprechen vorgeschriebenen Weise und in Annahme des Versprechens tätig geworden ist; die Auslobung hatte also - anders als heute nach $\S 657$ BGB - vertragliche Natur ${ }^{27}$. Es liegt auf der Hand,

26 Jurisprudenz Fall XVIII Nr. 24, S. 113f. (als Fall VII Nr. 24 noch in der 15. Aufl. 1927, S. 24). Der Herausgeber Detmold wurde vermutlich durch den Oxforder Zivilrechtsprofessor Goudy, der die 'Jurisprudenz' ins Englische übersetzt hatte (Oxford 1904; vgl. Vorwort der 13. Aufl.), auf den Fall hingewiesen: Herne Bay Steamboat Company v. Hutton, [1903] 2 Law Reports, King's Division 683 (Court of Appeal), dazu kurz Zimmermann (o. Fn. 18), S. 141 Fn. 110.

27 B.Windscheid, Th. Kipp, Lehrbuch des Pandektenrechts, 9. Aufl. Frankfurt/M. 1906, Bd. 2, S. 257 ff.; Jhering (o. Fn. 9), S. 93ff. mit C.F.F. Sintenis, Das prakt. Gemeine Civilrecht, Bd. 2, § 96 Fn. 58 (in 3. Aufl., Leipzig 1868, S. 283f.). 


\section{R. KNÜTEL}

dass der Mildtäter (= M) sich nicht gegenüber den Kindern verpflichten, sondern ihnen lediglich eine Erwerbschance eröffnen wollte. Eine Aneignung (Okkupation) würde an sich voraussetzen, dass $M$ das Eigentum an den versteckten Sachen aufgegeben und es damit auch Dritten ermöglicht hat, sie sich anzueignen. Da sich mit den Uhren auch einige wertvolle Sachen darunter befanden, ist das jedoch sehr zweifelhaft.

Näher zu liegen scheint deshalb, dass M Eigentumsübertragungen in incertas personas vorgenommen hat, wie das für das Auswerfen von Geschenken auf Umzügen in der Regel angenommen wird ${ }^{28}$. Da $\mathrm{M}$ diese Eigentumsübertragungen von Bedingungen abhängig machen kann, ist es ihm ohne weiteres möglich, den Kreis der personae incertae einzugrenzen, so dass das fremde Kind und der Arbeiter kein Eigentum an den Fundstücken erwerben könnten; den Arbeiter träfe zugleich eine vertragliche Herausgabepflicht.

In Betracht kommt aber auch die Lösung, dass M zwar die Sachen derelinquiert hat, jedoch nur zugunsten der Waisenkinder. Eine (bedingte) Eigentumsaufgabe zugunsten einer Aneignung durch bestimmte Dritte war im römischen Recht anerkannt und ist auch nach heutigem Recht möglich ${ }^{29}$. Ob Jhering auch diese Lösung in Betracht gezogen hat, ist zwar ungewiss, aber auch keineswegs fernliegend. Denn ihm mag bekannt gewesen sein, dass im Preußischen ALR ${ }^{30}$ die Dereliktion zur Aneignung durch Dritte ausdrücklich vorgesehen war: $\S 345$ I 9: "Hat jedoch jemand Sachen oder Gelder nur für gewisse Personen Preis gegeben, so können auch nur diese das Eigenthum durch Besitznehmung erwerben." Koch sah den Vorteil dieser Vorgehensweise darin, dass der Erwerb größere Bestandskraft habe. "Der Derelinquierende vollzieht dadurch keine Veräußerung im juristischen Sinne, mithin ist die darauf folgende (originäre) Erwerbung unanfechtbar." ${ }^{31}$ Selbstverständlich könnten auch bei dieser Konstruktion das fremde Kind und der Arbeiter nicht erwerben.

\section{3.}

Ein Streit um 80 Thlr. (Civilrechtsfälle 1847, Nr. XCVII; 1913 Nr. 67)

Der Fall ist weniger kompliziert, als man auf den ersten Blick vermuten möchte:

Der A hat gegen den X häufig einen Anspruch von 80 Thlr. geltend gemacht, den letzterer völlig bestreitet. Wie er wieder einmal den X wegen dieser Forderung anging, sagte letzterer: "Ich bin der Sache jetzt überdrüßig geworden und will lieber die 80 Thlr. zahlen, als Ihre alte Litanei immer von

28 Vgl. Windscheid, Kipp, 9. Aufl., Bd. 1, S. 888.

29 Näheres bei R. Knütel, Dereliktion zur Aneignung? in: W. Wiegand, Th. Koller, H.P. Walter (Hrsg.), Fschr. E. Bucher, Bern 2009, S. 351, 356 ff., 364 ff. Ein beachtlicher Unterschied ergibt sich etwa bei Sachen, die durch Drittrechte, z. B. einem Pfandrecht, belastet sind: Während bei der Übereignung an unbestimmte Personen ein gutgläubig lastenfreier Erwerb möglich ist ( $\$ 936$ Abs. 3 BGB), bleibt im Falle einer Dereliktion zur Aneignung das Pfandrecht (ungeachtet der zwischenzeitlichen Herrenlosigkeit) bestehen.

30 Vgl. z.B. Jhering, Der Besitzwille, Jena 1889, S. 347 Fn. 2 (348): "Obschon selber Romanist, sympathisire ich doch in diesem Punkt mehr mit dem preußischen, als mit römischen Recht...".

31 C.F. Koch, Allgemeines Landrecht für die Preuß. Staaten, Bd. 1, 6. Aufl., Berlin 1874, S. 463 Fn. 1. Weiteres bei Knütel (Fn. 29), S. 360 f. 


\section{RUDOLF VON JHERING UND DIE FREUDE AM RECHTSFALL}

neuem zu hören. Ich verspreche Ihnen hiermit, wenn Sie Ihrerseits die Grundlosigkeit Ihrer Ansprüche zugestehen und nie wieder weder gegen mich, noch gegen andere von unserem ganzen Schuldverhältniß ein Wort fallen und vor allem sich nicht wieder von einem Advokaten aufhetzen lassen wollen, die 80 Thlr. zu zahlen." A willigt seinerseits ein und acceptirt das Versprechen.

Da X später trotzdem nicht zahlt, so glaubt A, daß jener ihn angeführt hat. Denn, sagt er zu sich, schweige ich, so rührt X sich nicht; mahne und verklage ich ihn, so bekomme ich noch weniger etwas, denn meinen ehemaligen Anspruch habe ich aufgegeben und dafür ein Versprechen erhalten, dessen Wirksamkeit gerade davon abhängt, daß ich nicht klage.

Können wir dem A darauf etwas entgegnen?

Ungeachtet der Formulierung handelt es sich nicht um ein Schuldversprechen in Sinne des $\S 780$ BGB, das überdies nichtig wäre, weil es an der gebotenen Schriftform fehlt $(\S 125$ BGB). Vielmehr haben A und X, da das BGB insoweit dem früheren Recht folgt ${ }^{32}$, sowohl nach gemeinem Recht wie nach heutigem gemäß § 779 BGB einen Vergleich abgeschlossen, indem sie den Streit über ein Rechtsverhältnis, nämlich das Bestehen der Forderung, im Wege gegenseitigen Nachgebens beseitigten: A hat die zuvor bestrittene Forderung anerkannt und X sich auf deren Einschränkung durch die Bedingung eingelassen. Der Einhaltung einer Form bedurfte es dafür nicht.

Die Schwierigkeit des Falles liegt darin, dass A die Zahlungsklage aus der (durch den Vergleich begründeten) Forderung erfolgreich erst dann anstrengen könnte, wenn feststeht, dass er nie wieder von dem Schuldverhältnis ein Wort hat fallen lassen. Das aber ist erst im Zeitpunkt seines Todes klar. Das Problem ist uralt; es ergibt sich immer, wenn ein Erwerb unter der negativen Potestativbedingung erfolgt, dass der Erwerber etwas Bestimmtes nicht tun wird. Dazu hat der spätrepublikanische Jurist Quintus Mucius Scaevola ${ }^{33}$ die Lösung entwickelt, dass der Erwerber - etwa die mit einem Vermächtnis für den Fall bedachte Witwe, dass sie nicht wieder heiratet - die Zuwendung sogleich erhält, jedoch nur gegen das Sicherheitsversprechen, bei einer Zuwiderhandlung das Empfangene herauszugeben, die sog. cautio Muciana ${ }^{34}$. In $\S 2075$ BGB ist diese Lösung dahin fortentwickelt, dass die Zuwendung im Zweifel von der auflösenden Bedingung abhängig ist, dass der Bedachte die Handlung vornimmt. Der Bedachte kommt mithin schon zu Lebzeiten in den Genuss der Zuwendung und nicht erst sein Erbe.

Bei Erscheinen der "Civilrechtsfälle" 1847 war dem A folglich zu entgegnen, der Vergleich sei dahin auszulegen, dass er, A, von X Zahlung der 80 Thlr. gegen Sicherheitsleistung verlangen kann. Diese Auskunft bietet sich auch für die Zeit der letzten Auflage (1912) an ${ }^{35}$, weil eine analoge Anwendung der auf erbrechtliche Zuwendungen zugeschnittenen Regelung des $\S 2075$ methodisch schwerlich zu rechtfertigen ist.

32 Vgl. nur Windscheid, Kipp (Fn. 28), Bd. 2, S. 836.

33 Pontifex, geb. ca. 140 v. Chr., Konsul 95, ermordet 82.

34 Vgl. Pomp. D. 40,4,61 pr.

35 Zur Sicherheitsleistung vgl. §§ 232-240 BGB. 


\section{R. KNÜTEL}

4.4.

Das Gold in der Gans (Jurisprudenz 1889 Nr. XXI. 30; 1927 Nr. 29)

Einer Zeitung entnehme ich folgenden Fall, der an den Ring des Polykrates erinnert. In dem Magen einer von einer Händlerin gekauften Gans war neulich beim Schlachten ein Zwanzigmarkstück gefunden worden. Die Verkäuferin, welche durch Zufall Kenntniß davon erhalten hatte, kam alsbald zu dem Käufer und verlangte die Herausgabe des Goldstückes, das von der Gans verschluckt worden sei, so lange dieselbe noch in ihrem, der Händlerin, Besitz gewesen. Gleichzeitig erhob aber auch der Bauersmann, bei dem die unvermögende Händlerin zur Miethe wohnt, Einspruch gegen die Aushändigung des Fundstückes, weil er das Geldstück auf seinem Hofe verloren habe, während er seiner Mietherin gar nicht zutraute, überhaupt ein Zwanzigmarkstück besessen zu haben. Indeß auch der Käufer behauptet, Anspruch auf seinen Fund zu haben, da er mit der Gans alles, was sie in sich getragen, erworben habe.

Da die drei beteiligten Personen sich recht laienhaft äußern, ist von vornherein zu vermuten, dass keine das Wesentliche trifft ${ }^{36}$. Die Händlerin beruft sich auf einen früheren Besitz an der Gans. Doch war sie damit nicht zugleich Besitzerin des Goldstücks, von dem sie nichts wusste, wie ja auch der Eigentümer eines Grundstücks, in dem ein Schatz vergraben ist, von dem er nichts weiß, nicht Besitzer des Schatzes ist (vgl. Paul. D. 10,4,15; D. 41,2,3,3). Überdies hat die Händlerin den Besitz und das Eigentum an der Gans willentlich auf den Käufer (= K) übertragen, so dass schon deshalb Ansprüche wegen Besitzentziehung, aus früherem Besitz oder aus Eigentum ausscheiden ${ }^{37}$.

Dem Bauern wird es kaum gelingen, sein Eigentum an dem Goldstïck zu beweisen, und wenn er es könnte, bliebe ein gutgläubiger Eigentumserwerb des Käufers (=K) nach $\S \S$ 929, 932 BGB zu erwägen, ohne dass es auf das Abhandenkommen des Stücks ankäme, weil es sich um Geld handelt ( $\$ 935$ Abs. 2 BGB). In concreto ist dies allerdings nicht der Fall.

K. hat Eigentum an der Gans erworben, damit aber nicht zugleich am Goldstück, das nicht etwa Bestandteil oder Zubehör geworden, sondern eine selbständige Sache geblieben ist, von welcher K. beim Erwerb der Sache nichts wusste. Als K. das Goldstück im Magen der Gans entdeckte, war dies kein Schatzfund; denn "Schatz" kann nur eine Sache sein, "die so lange verborgen gelegen hat, dass der Eigentümer nicht mehr zu ermitteln ist" ${ }^{38}$. Diese "Eigenschaft" hat das Zwanzigmarkstück nicht. Sie ist aber entscheidend, weil zum einen

36 Wie Jhering bekennt, hat er bisweilen die Auffindung "des richtigen Gesichtspunktes durch eine verkehrte Begründung desselben ... zu erschweren gesucht", Civilrechtsfälle 1847, S. XIII.

37 Vgl. $\S \S 861,1007$ und $\S 985$ BGB. - Auch kommt eine Anfechtung der Verkaufserklärung wegen Eigenschaftsirrtums ( $\$ 119$ Abs. 2 BGB) nicht in Betracht, vgl. R. Knütel, Von Landanschwemmungen und Schätzen, in: G. Baumgärtel, H.J. Becker, E. Klingmüller, A. Wacke (Hrsg.), Fschr. H. Hübner, Berlin, New York 1984, S. 562, 569 (zum Schatz in der Kaufsache).

$38 \S 984$ BGB, vgl. Paul. D. 41,1,31,1: Thensaurus est vetus quaedam depositio pecuniae, cuius non exstat memoria, ut iam dominum non habeat ... 


\section{RUDOLF VON JHERING UND DIE FREUDE AM RECHTSFALL}

sie es erlaubt, die Sache als "quasi herrenlos" und damit als Gegenstand möglicher Aneignung (Okkupation) anzusehen. Zum anderen ist der Schatz eine Art Nebensache (accessio), die dem Rechtsschicksal der Hauptsache (des Grundstücks) zu folgen pflegt (accessio cedit principali). Die Regelung des Schatzfundes beruht also auf einer Kollision zweier gleichwertiger Prinzipien, des Okkupations- und des Akzessionsprinzips, die beide dieselbe Schwäche aufweisen, dass es sich in Wirklichkeit nicht um meine herrenlose und auch nicht um eine Sache handelt, deren Rechtsschicksal mit dem der Hauptsache verbunden ist. Da beide Prinzipien als gleichwertig erscheinen und beide dieselbe Schwäche aufweisen, empfahl es sich für den Kaiser Hadrian, die media sententia zu treffen, dass der Schatz zwischen dem Entdecker und dem Eigentümer der Mutter- oder Hauptsache aufzuteilen ist ${ }^{39}$. Dem folgt das BGB in $\S 984$.

Dem Schatzfund gegenüber steht der einfache Fund der $\S \S 965 f f$. BGB, den als Institut das römische Recht nicht kannte. Die Fundregelungen beruhen auf dem Bestreben, dem Verlierer, Eigentümer oder sonstigen Berechtigten die verlorene Sache nach Möglichkeit wieder zu verschaffen; sie begründen deshalb für den Finder ein besonderes Geschäftsbesorgungsverhältnis. Im Gegensatz zum Schatzfund setzt das Fundrecht gerade voraus, dass es möglich erscheint, den Eigentümer oder den sonst berechtigten Verlierer zu ermitteln. Auf dieser Grundlage ergibt sich, dass K. "Finder" des Goldstücks ist; er hat, wenn ihm der Empfangsberechtigte nicht bekannt ist, den Fund der zuständigen Behörde anzuzeigen; er kann grundsätzlich einen Finderlohn beanspruchen ( 971 BGB) und, wenn binnen bestimmter Frist kein Empfangsberechtigter seine Rechts angemeldet hat, am Ende auch das Eigentum am Goldstück erwerben.

Erinnerte Jhering sich bei dem Fall an den Ring des Polykrates ${ }^{40}$, so lässt sich aus der jüngeren Vergangenheit auch der Fall des niederländischen Hochseeanglers anführen ${ }^{41}$. Ihm war bei Windstärke 9 schlecht geworden und als er sich an der Reling übergeben musste, verlor er zugleich sein künstliches Gebiss. Nach knapp drei Monaten berichteten die Zeitungen, ein Angler aus Amsterdam habe im Magen eines 19 Pfund schweren Kabeljaus ein Gebiss gefunden. Es war das des Hochseeanglers, und es ist offenbar von niemandem bezweifelt worden, dass er Eigentümer geblieben war.

39 Vgl. nur M. Kaser, R. Knütel, S. Lohsse, Römisches Privatrecht, 21. Aufl. 2017, S. 157 Rz. 5 . Den o. Fn. 14 angeführten Autoren ist diese klare Grundlage des $§ 984$ BGB offenbar unbekannt.

40 Dieser - zunächst Kaufmann - hatte sich ca. 538 v. Chr. zum Tyrann von Samos aufgeschwungen, die Wirtschaft der Insel hochgebracht, die seinerzeit mächtigste Flotte bauen lassen und eine prächtige Hofhaltung geführt. Sein sagenhaftes Glück soll sich auch daran gezeigt haben, dass ein von ihm ins Meer geworfener höchst wertvoller Ring sich nach wenigen Tagen im Magen eines großen Fisches fand, den der Fischer ihm verehrt hatte. Das Glück endete, als er aufgrund einer List in die Hände des Satrapen Oroites fiel, der ihn in Magnesia am Mäander 522 umbringen und dann ans Kreuz schlagen ließ, Herodot III, 40-43; 120-126.

41 Berichtet in der Amsterdamer Zeitung "De Telegraaf" v. Mittwoch, d. 30. Nov. 1994, und in "Frankfurter Allgemeine Zeitung" v. 1. Dez. 1994, S. 11. 


\title{
R. KNÜTEL
}

4.5.

Der Streit um einen abgeschleppten Wal

(Civilrechtsfälle 1847 Nr. XCV; 1892 Nr. XCV, nicht mehr in 12. Aufl.)

\begin{abstract}
An der holländischen Küste trieb in den dreißiger Jahren unseres Jahrhunderts ein todter Walfisch an und ward von zwei verschiedenen Punkten aus zu gleicher Zeit bemerkt. Von beiden Punkten steuerten zwei Boote mit Fischern aus, um sich des Walfisches zu bemächtigen. Sie langten zu derselben Zeit, aber von gerade entgegengesetzter Seite bei demselben an, und die Fischer bestiegen ihn zugleich von zwei Seiten und kamen in der Mitte zusammen, wo sich ein Streit zwischen ihnen entspann. Währenddessen kam ein drittes Boot, und die Fischer aus demselben stellten den Streitenden vor, sie sollten den Streit auf dem Lande entweder fortsetzen oder vergleichsweise oder gerichtlich ausmachen; sie, die Neuangekommenen, wollten den Walfisch inzwischen an's Land bringen. Dieser Rath ward befolgt, und die Fischer des dritten Bootes zogen den Walfisch an's Land. Als nun die andern, die sich inzwischen verglichen hatten, den Fisch für sich in Anspruch nahmen, verweigerten die jetzigen Besitzer die Herausgabe desselben, und ihr Advocat verfocht ihre Sache mit folgenden Gründen, die einer Prüfung unterworfen werden sollen. Wir wollen der Kürze wegen die Fischer aus den beiden ersten Booten als zwei einzelne Personen, A und B, betrachten. Der Advocat der Beklagten behauptet nun, daß seine Klienten das Eigenthum des Walfisches durch Occupation erworben hätten, indem sie an dieser res nullius zuerst den Besitz erlangt hätten... ${ }^{42}$
\end{abstract}

Die Hauptfrage ist, ob A und B vor dem Eintreffen der Fischer im dritten Boot Besitz und Eigentum am Wal erlangt hatten ${ }^{43}$. Da sie beide gleichzeitig bei diesem eintrafen und ihn beide mit Besitz- und Aneignungswillen bestiegen, könnten sie Mitbesitz begründet haben. Das war im gemeinen Recht allerdings aus mehreren Gesichtspunkten sehr problematisch, insbesondere wegen des Paulus-Textes D. 41,2,3,5 (plures autem eandem rem in solidum possidere non possunt) mit der Veranschaulichung: "Denselben Besitz können ebensowenig zwei [jeder für sich allein] haben wie von Dir nicht angenommen werden kann, dass du auf derselben Stelle stehst, auf der ich stehe, oder dass du auf demselben Platz sitzt, auf dem ich sitze". Das versteht sich von selbst; ob man von daher (mit Labeo-Paul. D. 41,2,1 pr.) aber auch den Besitz überhaupt im Sinne einer faktischen und ausschließlichen Sachherrschaft verstehen muss, ist eine andere Frage. Fasst man den Begriff hingegen funktionaler

42 Die einzelnen Argumente der Advokaten können beiseite bleiben, weil sie im wesentlichen berücksichtigt werden.

43 Ein Besitz ist nicht etwa von vornherein dadurch ausgeschlossen, dass A und B nicht in der Lage waren, den Wal zu steuern. Man denke etwa an den Besitzer einer schwimmenden Insel oder an den kuriosen Fall aus der New York Times v. 12. Jan. 1930: Ein Jäger hatte einen Hirsch angeschossen und am Schwanz gepackt. Er wurde von dem Hirsch ca. $100 \mathrm{~m}$ mitgeschleift, als ein anderer Jäger den Hirsch erlegte und unter Protest des ersten an sich nahm. Der Mitgeschleifte war Besitzer. S. den Bericht von G. Kegel, Von wilden Tieren etc., in: H.C. Ficker, D. König u.a. (Hrsg.), Fschr. E. v. Caemmerer, Tübingen 1978, S. 149, 153. 


\section{RUDOLF VON JHERING UND DIE FREUDE AM RECHTSFALL}

auf, so kann man einen Doppelbesitz anerkennen ${ }^{44}$ und weiterhin auch einen Mitbesitz ${ }^{45}$. Dass beim Erwerb eines solchen Besitzes schon die Anteile der Mitbesitzer feststehen und ihnen bekannt sein müssten (Pomp. D. 41,3,32,2), wurde zunehmend in Frage gestellt ${ }^{46}$, und das Reichsgericht hat es 1885 für das gemeine Recht als zutreffend angesehen, dass ein auf einen ideellen Teil einer Sache beschränkter Besitz unmöglich sei ${ }^{47}$.

In $\S 866$ BGB ist dann, weil es Miteigentum gebe, auch der Mitbesitz anerkannt worden, sofern die Beteiligten Mitbesitzwillen haben ${ }^{48}$.

Einer Vertiefung bedarf es hier nicht; klar ist jedenfalls, dass Jherings Fall in dieser Frage seinen Jüngern des Rechts beachtliche Schwierigkeiten bereitet haben wird, die im Lauf der Zeit mit den Wandlungen des Besitzbegriffs freilich geringer wurden, Wandlungen, die auch in dem von Jhering mit Nachdruck verfochtenen Besitzbegriff hervortreten, welcher an das Moment anknüpft, worauf allein die Bedeutung des Besitzes beruhe, an das wirtschaftliche: "Besitz ist das thatsächliche Verhältniß der Person zur Sache, welches durch den Zweck ihrer wirtschaftlichen Verwendung geboten ist"49 und deshalb bei beweglichen Sachen, umzäunten und offenen Grundstücken etc. besteht und entsprechend ungleichen Anforderungen unterliegt.

Fraglich ist dann, ob die zwei Personen A und B überhaupt Mitbesitz erwerben können, wenn jede von ihnen den Alleinbesitz erlangen will ${ }^{50}$. Dem ließe sich entgegenhalten, dass A und B jedenfalls insoweit gemeinsame Sache machen würden, als es um die Abwehr Dritter geht, die den Fisch okkupieren wollen. Man wird auch nicht sagen können, A allein hätte den Besitz nicht erworben, weil $\mathrm{B}$, und $\mathrm{B}$ nicht, weil $\mathrm{A}$ ihm im Wege gestanden hätte (so der Advokat). Denn es wäre ganz lebensfremd, wenn eine Sache, die Platz für mehrere bietet, besitzlos sein sollte, obwohl sich zwei Okkupationsprätendenten auf ihr befinden. Wenn nicht von Mitbesitzern, so ließe sich wohl von Nebenbesitzern sprechen, von denen jeder nach außen Besitzschutz genießt, mögen sie auch untereinander die Besitzlage nicht geklärt haben. Da A und B sich jedoch inzwischen, also während der Bergung des Wals,

44 So Pomp. D. 43,26,15,4: Neben dem Interdiktenbesitz des Prekaristen verbleibe dem precario dans die Besitzposition, der es für die Ersitzung bedarf, so dass ein Doppelbesitz mit funktional geteilten Berechtigungen besteht, vgl. nur M. Kaser, R. Knütel, S. Lohsse, Römisches Privatrecht, 21. Aufl. München 2017, S. 124 Rz. 14.

45 Vgl. Cels. D. 13,6,5,15 (aufgeteilter Mitbesitz), Ulp. D. 43,17,1,7.

46 Vgl. H. Dernburg, Lehrbuch des Preuß. Privatrechts, Bd. I (Die Allgemeinen Lehren und das Sachenrecht), Halle 1884, S. 361f. Fn. 12.

47 "Der Mitbesitz Mehrerer an derselben Sache muß sowohl im natürlichen als auch im juristischen Sinne des Besitzes in der Weise aufgefaßt werden, daß ein jeder von ihnen die ganze Sache besitzt, jedoch in seinem Besitze durch den gleichen der übrigen beschränkt ist" (RGZ 13, S. 173, 179, Urt. v. 6. Feb. 1885).

48 Dass D. 41,2,3,5 keine Geltung mehr hat, merken F. Endemann, Lehrbuch des Bürgerl. Rechts, Bd. II/1, Berlin 1905, S. 161 Fn. 7; 216 Fn. 16 und E. Brodmann in: Planck, Kommentar z. BGB, Bd. III/1, 5. Aufl. Berlin 1933, § 866 Anm. 1 (S. 78) an.

49 R. v. Jhering, Der Besitzwille, Jena 1889, S. 481 u.ö.

50 Bedenken bei A. Pernice, Labeo, Bd. II/1 (C), 2. Aufl., Halle 1895, S. 415; F. Horak, Rationes decidendi, Aalen 1969, S. 285 Fn. 28. 


\section{R. KNÜTEL}

verglichen hatten, waren sie jedenfalls von da ab Mitbesitzer und aufgrund ihres Aneignungswillens Miteigentümer geworden. Auch hatten A und B ihren Besitz am Wal weder dadurch aufgegeben noch verloren, dass sie den Fischern ihr Einverständnis mit der Bergung bekundeten. Der Vorschlag der Fischer, A und B sollten ihren Streit auf dem Land klären, setzte voraus, dass es an Land überhaupt noch etwas zu klären gab, und ist deshalb als Anerkennung des Besitzes der beiden zu verstehen. Die Fischer des dritten Bootes konnten sich den nicht länger herrenlosen Wal mithin nicht aneignen. Allerdings steht diese Lösung unter dem Vorbehalt gewohnheitsrechtlicher Besonderheiten beim Walfang ${ }^{51}$.

V.

\section{1.}

$\mathrm{Zu}$ dem Göttinger Symposium "Jherings Erbe" aus Anlass der 150. Wiederkehr des Geburtstags steuerte Ernst Eduard Hirsch eine beachtliche Würdigung von "Jhering als Reformator des Rechtsunterrichts" bei ${ }^{52}$. Gleich zu Anfang hob er hervor, "daß die wichtigste Reform des Rechtsunterrichts im Gesamtbereich des kontinentalen Rechtskreises im Wintersemester 1854/55 an der Juristischen Fakultät Gießen von Jhering eingeleitet worden ist und sich dank der Ausstrahlungskraft seiner Persönlichkeit im Laufe von etwa vier Jahrzehnten in Deutschland und Österreich durchgesetzt hat: nämlich die Einführung praktischer Übungen (mit oder ohne schriftliche Arbeiten)." Das lässt sich in der Tat konstatieren $^{53}$, worauf noch kurz einzugehen ist, dürfte allerdings dahin zu korrigieren sein, dass die Anfänge schon nach Rostock in das Sommersemester $1847 \mathrm{zu}$ verlegen sind. Damals hielt Jhering sein zweites "Pandekten-Praktikum" und hatte mit 41 Teilnehmern den

51 So kam es, als Mitte des 18. Jahrhunderts die Engländer führende Walfangnation wurden, unter Verdrängung der gemeinrechtlichen Regelungen (Inst. 2,1,12; Gai. D. 41,1,5 pr.) zu einer engeren fast-fish rule, wonach ein Walfänger nur dann Besitz an einem Wal hatte, wenn er irgendeine unmittelbare ("feste") Verbindung zu ihm hatte, sei es durch Seil, Stab oder Stecken oder durch einen Mann aus der crew am oder auf dem Tier, s. dazu jüngst Chr. Hattenhauer, Fast-Fish and Loose-Fish. Die Konkurrenz um den Wal in Melvilles 'Moby Dick', in P. Spieß, Chr. Hattenhauer, M. Hettinger (Hrsg.), Homo heidelbergensis, Fschr. K.-P. Schroeder, Neustadt/Weinstr. 2017, S. 187, 197ff., der auf S. 198 Fn. 77 eine "Anekdote" zu einem Vorfall berichtet, der sich zugetragen haben kann: Zwei Walfangschiffe entdeckten einen Walkadaver. Ein Mitglied der crew des einen sprang ins Wasser, ergriff die Flosse des Wals und nahm ihn für sein Schiff in Beschlag. Der Kapitän des anderen Walfängers ließ sich heranrudern, sprach ihn an und fragte, ob es ihm (der den aufgeblähten Wal nicht zu besteigen vermochte) im Wasser nicht sehr kalt sei. Nach der bejahenden Antwort hatte man ihm gerade ins Boot geholfen, als der Kapitän seine Harpune in den Wal warf, seine Flagge setzte und ihn für sich reklamierte.

52 S. o. Fn. 6, S. 89-100. - Hirsch (1902-1985) war zuletzt Professor für Handelsrecht und Rechtssoziologie an der Freien Universität Berlin.

53 Vgl. auch Chr. Helfer, Rudolf von Jhering über das Rechtsstudium, Juristenzeitung (JZ) 21 (1966) 506f. - Zu Jherings Arbeit im Recht und seine Bedeutung für die Rechtstheorie des 20. Jh., auch in den USA, jüngst N. Jansen, M. Reimann, Begriff und Zweck in der Jurisprudenz - Ein Geburtstagsblatt für Rudolf von Jhering, ZEuP 25 (2018) 89ff. 


\section{RUDOLF VON JHERING UND DIE FREUDE AM RECHTSFALL}

größten Zulauf ${ }^{54}$. Die Civilrechtsfälle von 1847 müssen bereits vorgelegen haben; das Vorwort datiert vom November 1846, und das Heft wird (ebenso wie die zu vermutende Mundpropaganda) durch Jherings erstes Pandekten-Praktikum im Sommer 1846 veranlasst worden sein.

\section{2.}

Hirsch notiert die Civilrechtsfälle nur am Rande und betrachtet hauptsächlich die 'Jurisprudenz des täglichen Lebens' in Darstellung, Veränderung und Anpassung an Regelungen und Sprache des BGB. Er stellt noch für die letzte Auflage (v. 1927) fest, es sei "nicht das tägliche Leben schlechthin, das den Fällen die besondere Note gibt; es ist das tägliche Leben eines in guten Einkommens- und Vermögensverhältnissen lebenden, gern reisenden, den Tafelfreuden ergebenen, musikliebenden und selbst ausübenden, hoch gebildeten, nationalliberalen Bourgeois", dem Marx und Lasalle unbekannt geblieben seien (S. 95), und weiter: "Der Sachverhalt zahlreicher Fälle entspricht zwar dem damaligen täglichen Leben, ist aber für den Studenten unserer Zeit ebenso unwirklich, wie es zahlreiche Sachverhalte des Corpus Juris für den Studenten vor 100 Jahren gewesen sind" (S. 96). Hirsch bietet dazu auf zwei abgedruckten Seiten Kostproben aus der 5. und der 15. Auflage an.

Dem Anliegen Jherings wird das jedoch nicht gerecht, zumal da Hirsch die juristischen Gehalte der 'Jurisprudenz' völlig außer Betracht lässt. Wie schon oben (III.3) zur Omnibusfahrt oder zum Nachbarrecht deutlich wurde, geht es ganz überwiegend darum, durch knappe Schilderung und Abwandlungen einer Situation die Kenntnis von etablierten Lösungen des gemeinen Rechts (und später ent- oder widersprechenden des BGB) zu vermitteln ${ }^{55}$. Das Ziel der 'Jurisprudenz' ist vornehmlich, bei den Teilnehmern Wissen zu schaffen oder zu erweitern, und dabei spielen die tatsächlichen Umstände der jeweiligen Fallschilderung, die zugunsten der Merkfähigkeit eher eigenartig ausfallen sollte, nur eine geringe Rolle. Auch wäre eine Berücksichtigung der - wie Hirsch einräumt, Jhering selbstverständlich bekannten - politischen und sozialen Probleme des 19. Jahrhunderts und des Phänomens der Massen für das von ihm verfolgte Anliegen kaum hilfreich.

\section{3 .}

Erst im Schlussabschnitt der 'Jurisprudenz' (XXI "Allerlei") weicht Jhering von der bis dahin befolgten Konzeption ab und bringt auch Fälle, die in die Civilrechtsfälle gepasst hätten ${ }^{56}$. Jedenfalls ist die Sammlung der Civilrechtsfälle die erkennbar anspruchsvollere.

54 M. Kunze, Rudolf von Jhering - ein Lebensbild, in: O. Behrends (Hrsg.), Rudolf von Jhering. Beiträge und Zeugnisse, 2. Aufl. Göttingen 1993, S. 14.

55 Zum Beispiel lernen die Hörer im knappen Abschn. XVIII (Eine Seefahrt) der 7. Aufl. 1889 unter anderem die Partenreederei, das Seedarlehen, das Umladungsverbot (Lab.-Paul. D. 14,2,10,1 mit dem früheren $\S 565$ HGB nebst Vorläufer im ADHGB v. 1861), die lex Rhodia de iactu, die actio tributoria und Große Haverei, die donatio mortis causa, remuneratorische Schenkung und Kommorientenpraesumtion kennen (Nr. 1; 5-6; 7; 8; 13; 20; 21; 22) oder etwa, dass "Auf der Straße" ein dem Menageriebesitzer entsprungener Tiger nicht herrenlos wird (XI Nr. $11 \mathrm{u} .12$ ).

Vgl. die erörterten Fälle vom ehemaligen Waisenknaben und dem Gold in der Gans. 


\section{R. KNÜTEL}

Sie ist für Fortgeschrittene gedacht, die mit ihren Kenntnissen den Rechtsstoff im Fall auffinden und daraus die Lösung entwickeln sollen.

Die Sachverhalte sind oft recht ungewöhnlich, gelegentlich könnte man sie, wie etwa den Meteoriteneinschlag in Nachbars Garten, für Phantasieprodukte halten. Doch trat schon bei unseren Fällen zutage, dass auch sehr Unwahrscheinliches sich wiederholt, sei es auch in Abwandlung. Auch bekundet Jhering selbst verschiedentlich, dass er den Fall der Zeitung entnommen hat. Jedenfalls zeigt sich, dass seine Fälle anschaulich, einprägsam und juristisch ergiebig sind und damit vorzüglichen Diskussionsstoff bieten. Ebendas war Jherings aus eigener Erfahrung gewonnenes Ziel.

Er war damit seiner Zeit voraus. Denn es steht fest, dass anschauliche Fälle und Beispiele uns zum Erwerb unseres Wissens, zu dessen Organisation und dessen Anwendung von besonderem Nutzen sind. Wie die Lernpsychologie herausgefunden hat, werden in der Alternative, dass Wissen methodisch entweder über die Anführung von Beispielen oder die Darlegung von Prinzipien vermittelt wird, im Regelfall deutlich die Beispiele bevorzugt; der menschliche Verstand spricht sogar dann stärker auf Beispiele an, wenn diese schwach, im Zusammenhang mit ihnen die Prinzipien dagegen gut dargestellt werden ${ }^{57}$. Offenbar hängt das damit zusammen, dass Beispiele unmittelbare Anschauung verschaffen und ein "kurzschlüssiges" Erfassen der zugrunde liegenden Regel, Struktur oder Problematik erlauben.

Wiederholt hat Jhering in den Vorworten der Auflagen seiner Civilrechtsfälle erklärt, in seinen Augen sei "eine Theorie, die nicht unausgesetzt aus der Quelle praktischer Anregungen schöpft, ... um nichts besser ..., als die Naturwissenschaften es zu jenen Zeiten waren, da sie, statt bei der Natur, bei Aristoteles und Plinius in die Lehre gingen. In dieser Überzeugung habe ich", sagt er, "nicht bloß für mich selber von jeher den größten Werth darauf gelegt, jede Gelegenheit, die sich mir darbot, zu suchen und zu benutzen, um meine theoretischen Kenntnisse am einzelnen Fall zu verwerthen und zu erproben ..." ${ }^{15}$. Und auch an anderer Stelle bekennt er: "Seit länger als vierzig Jahren halte ich ein solches Pandektenpraktikum, und ich kann nicht genug rühmen, wie sehr es mich gefördert hat; es ist mir dadurch zur zweiten Natur geworden, bei allen Rechtssätzen, Begriffen, Unterschieden mir ihre Anwendung an einem konkreten Fall zu veranschaulichen und sie daran die Probe bestehen zu lassen, kurz das abstrakte Denken durch das kasuistische zu kontrollieren." 59

57 Reimann, Lernprozesse beim Wissenserwerb aus Beispielen, 1997, S. 28f. (nach LeFevre/Dixon), vgl. R. Knütel, Von befreiten Vögeln [-] oder: Exempla docent, Juristische Schulung (JuS) 41 (2001) 209.

58 Civilrechtsfälle, 5. Aufl. 1888, S. IV; 6. Aufl. 1892, S. IV/V; 11. Aufl. 1909, S. V.

59 Jhering, Scherz und Ernst in der Jurisprudenz, 13. Aufl. Leipzig 1924 (Nachdruck Darmstadt 1980), S. 366 f. - Das entspricht der Interpretationsfunktion von Fällen und Beispielen, bei der es darum geht, (abstrakte) Begriffe durch Referenzbeispiele oder -fälle aufzuschlüsseln und damit ihre Reichweite zu kontrollieren. - Übrigens hätte ein römischer Jurist sich ähnlich wie Jhering ausdrücken können. 


\section{RUDOLF VON JHERING UND DIE FREUDE AM RECHTSFALL}

\section{4 .}

Darüber hinaus berichtet Jhering, schon seit seinem ersten Auftreten als Privatdozent habe er im Pandekten-Praktikum die Zuhörer zur schriftlichen Bearbeitung der Rechtsfälle veranlasst und sämtliche Arbeiten schriftlicher Korrektur unterzogen. In der Wiener Zeit habe er wegen der großen Masse von Zuhörern (bis nahe auf 200) den Ausweg gewählt, sie Sektionen bilden zu lassen, die sich die von ihren Mitgliedern verfassten Arbeiten anhören und durch Abstimmung die besten davon ermitteln sollten, die er dann durchgesehen und in der Vorlesung besprochen habe ${ }^{60}$. Und auch in Göttingen habe sich der höchst wirksame Einfluss des Pandekten-Praktikums auf die juristische Bildung bewährt ${ }^{61}$.

VI.

6.1.

Als Ferdinand Regelsberger 1897 die 8. Aufl. der Civilrechtsfälle herausgab, hob er in seiner Vorrede ${ }^{62}$ hervor, jahrelang habe Jhering dafür gekämpft, den theoretischen juristischen Unterricht durch die Übung in der Entscheidung von Rechtsfällen zu ergänzen, erst als nahezu einsamer Rufer im Streit, doch habe er dann mehr und mehr Anhänger und Nachfolge gefunden mit dem Ergebnis: "Die juristischen Praktika bilden heutzutage einen eisernen Bestand in der Lehrtätigkeit der deutschen Juristenfakultäten".

Aufschlussreich zu den Folgen von Jherings pädagogischem Wirken sind auch die Nachbemerkungen, die einer der einflussreichsten Rechtslehrer seiner Zeit, Ernst Zitelmann ${ }^{63}$, seiner Sammlung 'Rechtsfälle für bürgerrechtliche Übungen' ${ }^{\prime 64}$ beigegeben hat:

"Eine Pflicht tiefer Dankbarkeit erfülle ich, indem ich hier noch einmal Rudolph von Jherings erlauchten Namen nenne. Auf ihn gehen die Übungen, wie sie heute an allen Rechtsfakultäten veranstaltet werden, zurück. In den siebziger Jahren hielt er in Göttingen allsommerlich sein vielbewundertes Zivilrechts-Praktikum ... Jhering hingegen stellte die Rechtsfall-Übungen als ebenbürtige Unterrichtsart den theoretischen Vorlesungen zur Seite ... und erzwang ihnen durch seine hinreißende Persönlichkeit die allgemeinste Teilnahme ... Auf seinen Rat hielt ich schon in Göttingen neben anderen Übungen auch Rechtsfallübungen im Winter ab. Dann konnte ich diese Art des Unterrichts ... 1879 in Rostock und ebenso 1881 in Halle a.S. und 1884 in Bonn neu einführen. Die preußische Unterrichtsverwaltung ermaß die Wichtigkeit der Übungen vollständig und nahm ihre Veranstaltungen bereits in meine Hallische Lehrverpflichtung auf. Zugleich versuchte ich das Übungswesen reicher auszubauen."

60 Näheres zur Eröffnung und Organisation des Praktikums in Wien enthält ein Brief Jherings v. 25.10.1868 an Oskar Bülow; Auszug bei Hirsch, S. 90 (aus H. Ehrenberg, Rud. v. Jhering in Briefen an seine Freunde, Leipzig 1913, S. 225).

61 Civilrechtsfälle, 5. Aufl. 1888, S. V; 12. Aufl. 1913, S. VI.

62 S. IV.

63 1852-1923 (Römisches, Bürgerliches und Internationales Privatrecht).

64 München, Leipzig 1917, S. 202f. - Ausführlichere Wiedergabe bei Hirsch, S. 90f. 


\section{R. KNÜTEL}

Dies geschah dadurch, dass er die allgemeinen Übungen mit Rechtsfällen (also Jherings Pandekten-Praktikum) von den exegetischen Übungen trennte und für jüngere Studierende Anfängerübungen einführte. Nachdem er dies auch in Bonn durchgesetzt hatte, "sind diese Übungen Gemeingut aller Universitäten geworden". 65

Zitelmann beschließt seine Ausführungen mit einer Prophezeiung, die sich leider nicht verwirklicht hat: "Der Fortentwicklung der Übungen jeglicher Art unter gleichzeitiger Zusammendrängung der theoretischen Vorlesungen gehört die Zukunft des Rechtsunterrichts" 66 .

\section{2.}

Aus der Freude an der Lehre, dem Verantwortungsbewusstsein gegenüber den Studierenden und mit Sicherheit auch aus der Freude am Rechtsfall, insbesondere am eigenartigen und merkwürdigen, wird sich das wahrlich große und sein ganzes wissenschaftliches Leben andauernde und kräftezehrende Engagement Rudolf von Jherings für die juristische Ausbildung an Rechtsfällen erklären. Das (Pandekten-) Praktikum war seine allseits anerkannte pädagogische Glanzleistung ${ }^{67}$.

Gern liest man, welch' schöne Erfüllung ihm dies gegeben hat:

"Die Mühe, die ich darauf ${ }^{68}$ verwandt habe, halte ich für eine der am besten angewandten meines Lebens; ich kann mir kein schöneres Ziel meines ganzen Wirkens denken, als den demnächstigen Juristen schon auf der Universität innerlich für die Jurisprudenz zu gewinnen, und daß dies durch nichts so leicht und sicher geschehen kann als dadurch, daß man ihm dieselbe nicht bloß in ihrer abstrakten, sondern zugleich in einer konkreten Gestalt vorführt, die imstande ist, sein Interesse zu fesseln, ist eine Überzeugung, die sich bei mir auf eine mehr als 40jährige Erfahrung stützt." 69

Prof. Dr. Rolf Knütel ${ }^{70}$

Bonn

65 Zitelmann, S. 203. - Unzutreffend ist die Annahme Jan Schröders, Rudolf Stammler habe mit seinen praktischen Pandektenübungen "die modernen zivilrechtlichen Anfängerübungen begründet" (in: G. Kleinheyer, J. Schröder, Deutsche und Europäische Juristen aus neun Jahrhunderten, 5. Aufl. 2008, S. 404). Zudem dürften Stammlers Pandektenübungen (2. Aufl. Leipzig 1896, 536 [!] S.) Anfänger überfordert haben.

66 Demgegenüber sind die Übungen mittlerweile sehr zurückgeschnitten worden; die Hausarbeiten sind entfallen und vielerorts wird während des Studiums nur eine einzige verlangt.

67 Vgl. Kunze (Fn. 54), S. 18 u. - Wie er selbst dieses Praktikum erlebt hat, bringt er in Scherz und Ernst (o. Fn. 59) S. 367 zum Ausdruck, etwa mit dem Hinweis: "Er (sc. der Docent) muß herabsteigen von seiner Höhe und gewärtigen, daß die Rollen des Fragenden und Gefragten vertauscht, und daß ihm Fragen vorgelegt werden, auf die er nicht gefaßt war. Er muß nicht bloß über ein sicheres, exaktes Wissen gebieten, sondern er muß es in jedem Augenblick ohne einen großen Apparat ohne lange Deduktionen in knappster, präcisester Form auf die gerade vorliegende Frage zur Anwendung zu bringen verstehen...".

68 Nämlich die Darstellung der Rechtsfälle, was wir gewiß auf den damit verbundenen Unterricht erstrecken können.

69 Civilrechtsfälle, 8. Aufl. 1897, S. X; 12. Aufl. 1913, S. VII.

70 Prof. Dr. Rolf Knütel ist Professor emeritus (Römisches und Bürgerliches Recht) an der Rheinischen Friedrich-Wilhelms-Universität Bonn. 